\title{
BSONIQ: A 3-D EEG SOUND INSTALLATION
}

\author{
Marlene Mathew
}

\author{
New York University, \\ Music Technology \\ New York, NY USA \\ mm5351@nyu.edu
}

Mert Cetinkaya

\author{
New York University, \\ Music Technology \\ New York, NY USA \\ mc5993@nyu.edu
}

\author{
Agnieszka Roginska \\ New York University, \\ Music Technology \\ New York, NY USA \\ roginska@nyu.edu
}

\begin{abstract}
Brain Computer Interface (BCI) methods have received a lot of attention in the past several decades, owing to the exciting possibility of computer-aided communication with the outside world. Most BCIs allow users to control an external entity such as games, prosthetics, musical output etc. or are used for offline medical diagnosis processing. Most $\mathrm{BCIs}$ that provide neurofeedback, usually categorize the brainwaves into mental states for the user to interact with. Raw brainwave interaction by the user is not usually a feature that is readily available for a lot of popular BCIs. If there is, the user has to pay for or go through an additional process for raw brain wave data access and interaction.
\end{abstract}

BSoniq is a multi-channel interactive neurofeedback installation which, allows for real-time sonification and visualization of electroencephalogram (EEG) data. This EEG data provides multivariate information about human brain activity. Here, a multivariate event-based sonification is proposed using 3D spatial location to provide cues about these particular events. With BSoniq, users can listen to the various sounds (raw brain waves) emitted from their brain or parts of their brain and perceive their own brainwave activities in a 3D spatialized surrounding giving them a sense that they are inside their own heads.

\section{INTRODUCTION}

Sonification is the method of rendering sound in response to data and interactions and sets a clear focus on the use of sound to convey information [1]. Electroencephalogram (EEG) is the recording of electrical potential from the human scalp containing multivariate data. EEG sonification has been very useful in areas spanning data analysis and medical diagnosis to general-purpose user interfaces in car navigation systems [2]. EEG sonification can give researchers or medical professionals a better idea as to what is happening at a certain location in the brain, when visual analysis can no longer be applied. For example, with fMRI, visual images (scans) of the brain are taken every millisecond, where the analysis of the brain activity takes place after the scan. EEG sonification provides information about brain activity in real-time by providing auditory images that can more easily be interpreted due to their spatial differences [7]. This is one of the main advantages of auditory displays over visual displays. Listening is used as a means to perceive data. Audio feedback for positional control could be very useful in for example, the medical field [5]. Sound is a temporal indicator of the ongoing physical processes in the world around us [16].

This paper presents "BSoniq", a 3-D EEG sound installation, in which, the user can perceive spatial characteristics of EEG signals in a multi-channel environment. With this installation, the users (listeners) wear a wireless EEG headset and listen to sounds generated in real-time from their brain waves to perceive brain activities which they may not be aware of in their daily life. To accomplish a brain electrical activity sonification, brainwave source localization features of multichannel EEG are converted into sound images. These allow for simple interpretation, because of their spatial temporal differences. Signals recorded from the scalp are "decoded" from the multi-channel EEG, by applying filters and modulation to the EEG signal with an audio file. The main goal is to use sound to render the original data in a suitably transformed way so that we can invoke our natural pattern recognition capabilities to search for regularities and structures. Brainwave sonification is also very practical in brain-computer interface (BCI) user feedback design. Deciding how the control of parameters, processing and filtering of inaudible data are used is important in this process. Using listening as a tool serves both as an aesthetic and/or scientific purpose. The human hearing system is able to decode and interpret complex auditory scenes. The more structured the representation of the sonified data, the better the accessibility and intelligibility of the chosen process [9].

We propose to employ auditory feedback, and thus provide visualization of the brainwaves in the form of spatial sound images, that is, to perform sonification of brain electrical activity. The 14 channels used in this project represent the 14 sensors of the EEG device used.

\section{BACKGROUND}

Efficient perceptualization of biofeedback or medical data requires a multidisciplinary approach, including the fields of computer science, engineering, psychology and neurophysiology [5]. EEG provides a diagnostically important stream of multivariate data of the activity of the human brain. One of the first attempts of auditory EEG exploration was reported in 1934 by E. Adrian and B. Matthews [15]. They measured the brain activity from a human subject from 
electrodes that were applied to the head, and the channels connected to these electrodes were viewed optically on bromide paper while being directly transduced into sound.

T. Hermann et al. have presented different strategies of sonification for human EEG [3]. Baier et al. used multivariate sonification that displayed salient rhythms as well as used pitch and spatial location to provide cues [15]. Hunt and Hermann conducted experiments to explore interactive sonifications, which they describe as the discipline of data exploration by interactively manipulating the data's transformation into sound [16]. They also realized that the individuality of interacting with sound is important, meaning that one must be able to detect a particular signal even if there are other interfering signals and/or a noisy background present.

There are many experiments converting multi-channel EEG to sound. However, not many use 3D sound to provide spatial cues. Hori and Rutkowski developed an EEG installation, sonifying 14 EEG signals using 5 channels, where the loudspeakers were geometrically located surrounding the listener and termed "A" to "E" from the left to the right [2] on the azimuth angle. By using only five channels, multiple EEG data were combined into one, which was processed and sent to a loud-speaker. This does not allow for details of a specific sensor to be perceived. BSoniq sonifies all 14 channels to speakers located at the azimuth and elevation angles related to an EEG sensor's location for monitoring purposes.

The main areas of EEG sonification are: EEG monitoring, EEG Diagnostics, Neurofeedback, Brain Computer Interface(BCI) feedback and communication as well as EEG mapping to music [11]. BSoniq's main focus is on monitoring or listening. Monitoring generally requires the listener to attend to a sonification over a course of time, to detect events, and identify the meaning of the event in the context of the system's operation [13].

\section{HARDWARE}

The Emotiv EEG wireless device is used for signal acquisition in this installation. This device has 14 sensors based on the International 10-20 system located at AF3, F7, F3, FC5, T7, P7, O1, O2, P8, T8, FC6, F4, F8 and AF4 (Fig. 1) [14]. The International 10-20 system is an internationally recognized method used to describe the location of scalp electrodes and the underlying cerebral cortex [12]. It was created to ensure a standard format so that the studies of a subject's EEG could be compared over time and subjects could be compared to each other. The "10" and "20" refer to the actual distances between adjacent electrodes that are either $10 \%$ or $20 \%$ of the total front-back or right-left distance of the skull.

The transmitted wireless EEG signals are received by the Emotiv USB receiver which is connected to a USB port of a PC and sent to Max/MSP for transformation. The data stream coming from the Emotiv device is encrypted by proprietary software, which is subsequently decrypted by Emotiv's SDK. The data is transmitted via the Emotiv's API as raw EEG values in microvolts. The EEG data is then stored as floating point values which are converted from the unsigned 14-bit output from the headset [10].

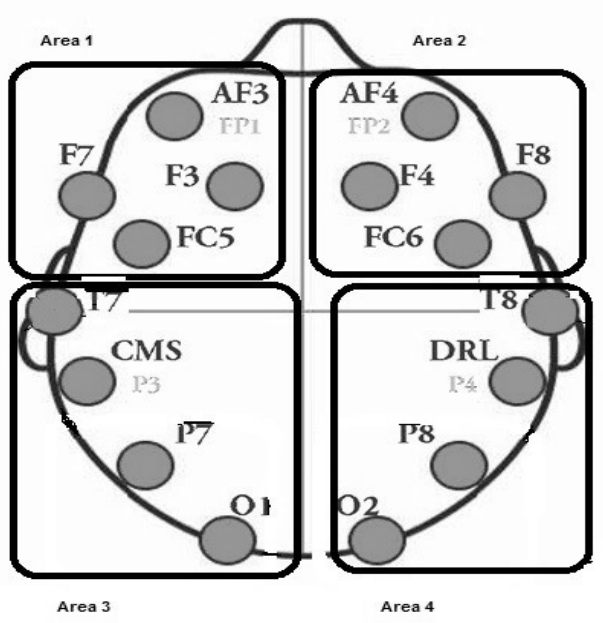

Figure 1: Area division of sensors

Once the EEG signals have been transformed, the sonified data are converted to analog audio signals using audio interfaces and sent to 14 speakers which are geometrically located around the listener. The layout of the speakers represents the layout of the sensors on the user's head, giving the impression that the user is inside his/her own head listening to the various brainwaves in action. The ring topology of the speakers is to provide cues of the azimuth and elevation in the horizontal plane. This, to focus the listener's attention to the correct angle of the sonified signal. Locations of the 14 loudspeakers used in this project are shown in Figure 4. A full sphere setup was used with ten loudspeakers positioned horizontally around the listener and the rest of the speakers were elevated approximately 40 degrees above the listener's head. Details of the sonification process is discussed in the following section.

\section{SOFTWARE}

Max/MSP, a visual programming language is used for EEG sonification. The actual EEG data transmission is based on Open Sound Control (OSC) protocol, provided by Mind Your OSCs. This is an open source software that sends the raw EEG values received from the Emotiv EEG SDK via User Datagram Protocol (UDP) to Max/MSP for transformation. The sonification is carried out sensor-wise with a sub-patch that receives a single channel EEG signal, which is band-limited and scaled to modulate a sample file. After the modulation takes place, the audio signal is sent via a single channel out to the loudspeaker. For example, the EEG signal of the AF3 channel after being transformed is sent to speaker 10. The full assignments of the EEG channels to the speakers are shown in Table 1. The sonified EEG signal of each electrode is sent to the speaker representing the general location of that electrode on the scalp. 


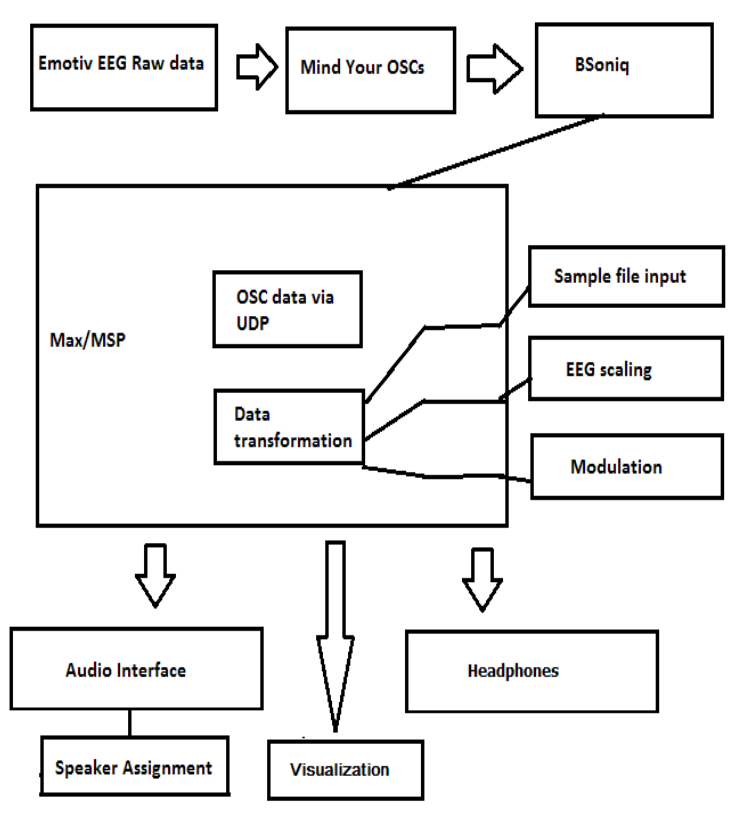

Figure 2: BSoniq flowchart

\subsection{Data Representation}

When dealing with sonification it is important to choose a specific sound dimension to represent a given data dimension[13]. EEG signals can range from 0.5 to $40 \mathrm{~Hz}$, which makes pitch a good sound attribute to represent any changes in the EEG data. Here, frequency modulation is used to transform the frequency of the EEG signal.

The EEG signal modulation used here is similar to that of a regular Frequency Modulation (FM) synthesis, where you have a carrier signal and a modulating signal. In this project the modulator is the EEG signal and the carrier is the looping sample file.

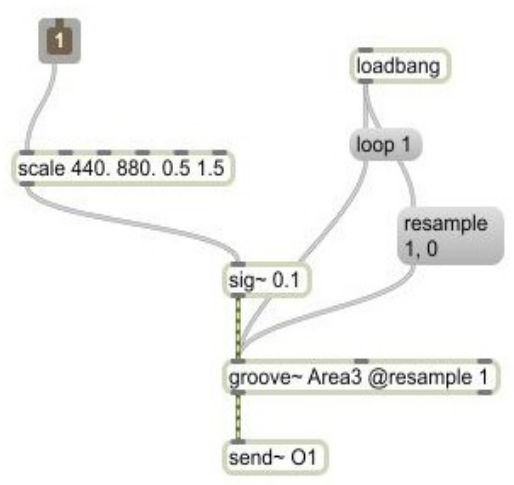

Figure 3: 'test' modulation patch
Table 1: Speaker Assignment

$\begin{array}{ll}\text { Sensor } & \text { Speaker } \\ \text { AF4 } & 1 \\ \text { F8 } & 2 \\ \text { T8 } & 3 \\ \text { P8 } & 4 \\ \text { O2 } & 5 \\ \text { O1 } & 6 \\ \text { P7 } & 7 \\ \text { T7 } & 8 \\ \text { F7 } & 9 \\ \text { AF3 } & 10 \\ \text { F3 } & 11 \\ \text { F4 } & 12 \\ \text { FC5 } & 13 \\ \text { FC6 } & 14\end{array}$

\subsection{Scaling}

Scaling is important to determine how much the pitch of a sound is used to convey a given change [13]. It shows the relationship between the system and the EEG data. Because each EEG signal may have different frequency and dynamic characteristics, the ability for the user to manipulate the scaling data is important for a better representation of the EEG data and its characteristics.

When the EEG signal is received by the Max/MSP program, it is first limited to $0-4500 \mathrm{uVolts}$ and then scaled from $0-0.5$. The scaled EEG signal is then sent to another subpatch 'test' (Fig. 3) to modulate it with a sample file. The sample file (carrier) is a looping audio file excerpt selected by the user. BSoniq provides the user with the flexibility as to what sounds (sample files) to choose for the sonification process, therefor enhancing the listening experience.

Since EEG signals typically range $0.5-40 \mathrm{~Hz}$, the data here has been scaled by default between $440-880 \mathrm{~Hz}$ following an octave music model. The user has the option to adjust the range. The data is scaled to values between 0.5 and 1.5 . These values determine the amplitude of the modulating signal. The default scaling translates higher EEG values into higher amplitude vectors, and lower EEG values into lower amplitude vectors, which has an effect on the sound output.

\subsection{Modulation}

At the end of dynamic scaling processes, the modulation process is applied. In BSoniq, the sensors are divided into four areas as shown in Figure 1. The user can choose a different sample file for each area or the same sample file for all four areas. The option for the user to select audio files, allows for a better distinction between the various brain activity levels at sensor level. After this process, the transformed EEG signal is sent to the corresponding loudspeaker. For example, in Fig. 3 the $\mathrm{O} 1$ sensor in Area 3 is sonified and sent to loudspeaker 6 . 


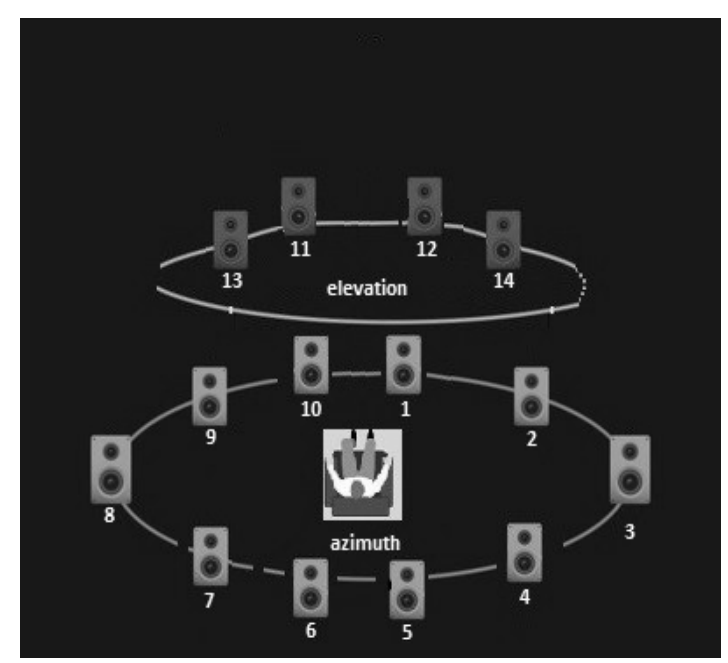

Figure 4: Speaker Layout

\subsection{Visual Representation}

Though the main feature of BSoniq is its auditory display, it can also visually display the relationship between the activity level of each EEG channel. This optional feature aids the user in visualizing what sensors are active and how much. The visualization is represented by a $3 \mathrm{D}$ model head as shown in Fig. 5 and 14 balls representing the EEG sensors and location. These balls, created in Max/MSP/Jitter uses the 'jit.gl.gridshape' object, which generates simple geometric shapes as a connected grid, in this case a sphere. These spherical shapes (balls) increase and decrease in size based on the sensor activity levels. The values used for visualization are the same scaled values used for sonification. The stronger the EEG signal the larger balls become. BSoniq also provides the user with several angles to turn the head model for a better view of the sensors. For example, if the user wants to have a better view of the back sensors, he/she can rotate the head for a side or back view of the balls (sensors).

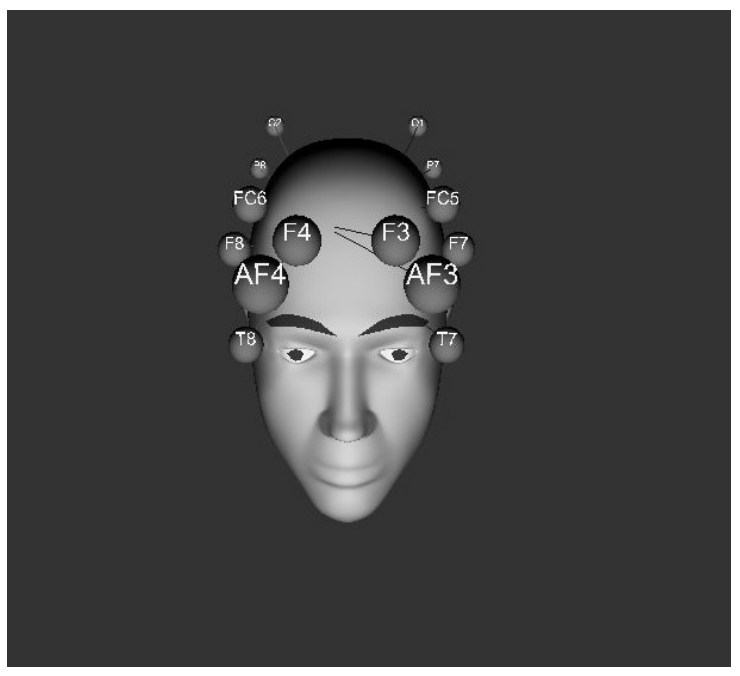

Figure 5: Visual representation of sensor activity

\subsection{Headphones}

As described in the previous sections, BSoniq was first designed as an installation for a 14-channel loudspeaker setup. However, most people do not have a 14-channel loudspeaker system accessible to them. Since this installation was intended for general use and the ability to convolve each modulated EEG signal with a corresponding loudspeaker impulse response (IR), brought on the idea of taking this project to the next level. Users would be able to experience BSoniq with a pair of headphones allowing flexibility for its use in nonlaboratory environments.

In the binaural format of BSoniq, the approach is to start with measuring impulse responses of each loudspeakers that are geometrically setup to represent the 14 sensor locations of the Emotiv EEG device using the Neumann KU-100 Dummy head. The resulting recorded stereo impulse responses are then split into left and right channels using Matlab, yielding a total of 28 impulse responses. Max/MSP is used here to provide the binaural experience in real-time.
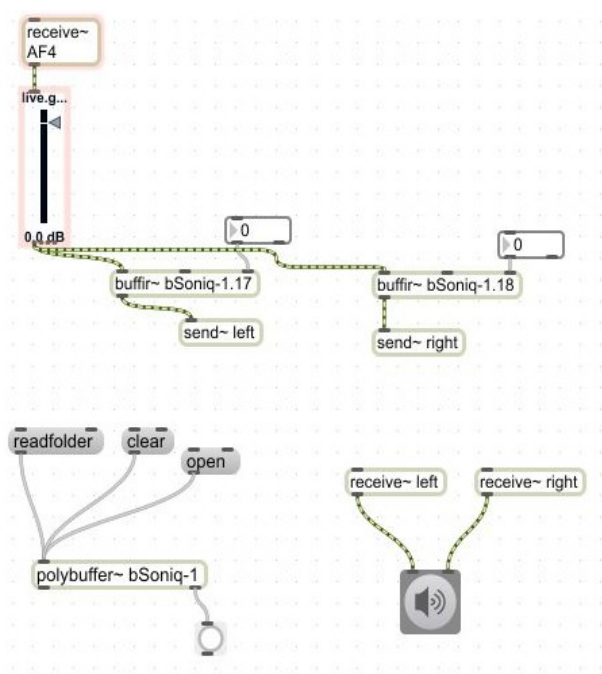

Figure 6: Partial HRTF patch

In Max/MSP, we used the "buffir $\sim$ " object, which is described as "a buffer-based FIR filter that convolves an input signal with samples from an input buffer" [16]. In this case the EEG signal is convolved with the corresponding IR. Since we are dealing with many channels of audio, we used the "polybuffer " object to ease the process of loading and delivering the IRs to corresponding "buffir $\sim$ " objects. After each EEG convolution, the output is sent to the corresponding left or right channel, thus giving a virtual representation of the various EEG sensor locations. To give an example, in Figure 6, the signal from the AF4 sensor is sent to the buffir $\sim$ object, which takes the left output signal and convolves it with IR sample 17 and convolving right output signal with IR sample 18. After the convolution of the AF4 signal, both left and right convolved signals are sent to the "ezdac $\sim$ " object for output. 


\section{PROTOTYPING}

The prototyping phase included evaluation by five users; three males and two females. Most users were able to distinguish EEG frequency changes as a result of the modulation process. Users did indicate that using a sample file of sound they were very familiar with, helped them to quicker understand the sonification process. For example, one user could not distinguish much difference with a sample file that was a bell sound, but was able to pick up on subtle frequency changes with a sound sample that was a snippet of a song he knew very well. While wearing headphones, users were also able to hear the location from where a particular modulated EEG signal was coming from, indicating the virtual placement of the sensors.

\section{DISCUSSION}

We presented BSoniq, which uses multi-channels to sonically represent EEG data in real-time 3D space using frequency modulation. BSoniq could be used for both online and offline sonification. By applying filters and parameter controls, it is possible for the user to focus on the area of interest within the signal. This is useful for real-time applications like EEG monitoring or EEG feedback. The inclusion of 360 degrees spatial cues permits the parallel sonification of many or all electrodes without losing clarity in the display. Clarity of the sonification, however also depends on the strength of the EEG signal capture from the device. The signal could also contain artifacts, which could be reduced or removed in order to yield a clearer signal for the display. What also needs to be noted is that the perceptual capabilities of the listener is important. If the listener is unable to distinguish sounds or incapable of hearing certain frequencies, then this would affect the user's perception of the installation's functionality.

Future work includes conducting additional evaluations for necessary design improvements as well as upgrading BSoniq to include other popular EEG devices. The current installation only allows for the user to remain stationary. Allowing the user's head movement and tracking, is a feature that will be added to create a fully integrated system.

To conclude, we believe we accomplished our goal of EEG sonification using 3D spatial cues. Even though BSoniq started out as an installation, mainly for an aesthetic user listening experience, we also believe that in addition to sonification, the visualization component could also be enhanced into an artistic EEG visualization application using geometric data and transformations for artistic applications. That is, exploring methods that uses OpenGL for example, to create 3D spatial-spectral representations of an EEG signal.

\section{REFERENCES}

[1] Thomas Hermann, Andy Hunt and John Neuhoff. "Auditory Display and Sonification". The Sonification Handbook, 2011.

[2] Gen Hori and Tomasz M. Rutkowski. "Brain listening-a sound installation with EEG sonification". Journal of the Japanese Society for Sonic Arts, 4(3):4-7, 2000.
[3] Thomas Hermann and Helge Ritter. "Listen to your data: Model-based sonification for data analysis". Advances in intelligent computing and multimedia systems, 8:189-194, 1999.

[4] Stephen Barrass and Gregory Kramer. "Using sonification". Multimedia systems, 7(1):23-31, 1999.

[5] Emil Jovanov, Dusan Starcevic, and Vlada Radivojevic. "Perceptualization of biomedical data". IN MEDICINE, page 189, 2001.

[6] Teruaki Kaniwa, Hiroko Terasawa, Masaki Matsubara, Tomasz M Rutkowski, and Shoji Makino. "EEG auditory steady-state synchrony patterns sonification". In Signal \& Information Processing Association Annual Summit and Conference (APSIPA ASC), 2012 Asia-Pacific, pages 1-6. IEEE, 2012.

[7] Tomasz M Rutkowski. "Multichannel EEG sonification with ambisonics spatial sound environment". In Asia-Pacific Signal and Information Processing Association, 2014 Annual Summit and Conference (APSIPA), pages 1-4. IEEE, 2014.

[8] Tomasz M Rutkowski, Francois Vialatte, Andrzej Cichocki, Danilo P Mandic, and Allan Kardec Barros. "Auditory feedback for brain computer interface managementan EEG data sonification approach". In Knowledge-Based Intelligent Information and Engineering Systems, pages 12321239. Springer, 2006.

[9] Timothy Schmele and Imanol Gomez. "Exploring 3d audio for brain sonification". In International Conference of Auditory Display, 2012.

[10] Haracio Tome-Marques and Bruce Pennycook. "From the unseen to the s[cr]een eshofuni, an approach towards real-time representation of brain data". 2014.

[11] A Väljamäe, T Steffert, S Holland, X Marimon, R Benitez, S Mealla, A Oliveira, and S Jordà. "A review of realtime EEG sonification research". In International Conference of Auditory Display, 2013.

[12] Neuroscience For Kids. (n.d.)., from $<$ http://faculty.washington.edu/chudler/1020.html $>$ Retrieved February 10, 2016.

[13] Walker, Bruce, and Nees, Michael. "Theory of sonification."The Sonification Handbook: 9-39, 2011.

[14] Emotiv Epoc EEG. <https://www.emotiv.com>. Retrieved July 13, 2015.

[15] Gerold Baier, Thomas Hermann, and Ulrich Stephani. "Multi-channel sonification of human EEG". In Proceedings of the 13th International Conference on Auditory Display, 2007.

[16] Max/MSP/Jitter Graphic software development environment. Cycling '74. <www.cycling74.com>. Retrieved November 3, 2016.

\section{(1) (3)}

This work is licensed under Creative Commons Attribution - Non Commercial 4.0 International License. The full terms of the License are available at http://creativecommons.org/licenses/by-nc/4.0/ 